\title{
TEMPERATURAS DECADAIS EXTREMAS E MÉdIA EM SÃO BENTO DO UNA- PERNAMBUCO, BRASIL
}

\section{EXTREME AVERAGE DECADAL TEMPERATURES IN SÃO BENTO DO UNA - PERNAMBUCO, BRAZIL}

Raimundo Mainar de Medeiros ${ }^{1}$, Luciano Marcelo Falle Saboya ${ }^{2}$, Manoel Vieira de França ${ }^{3}$, Romildo Morant de Holanda $^{4}$, Fernando Cartaxo Rolim Neto ${ }^{5}$, Wagner Rodolfo de Araújo ${ }^{6}$, João Carlos Montenegro Coutinho Junior ${ }^{7}$

Submetido em: 07/09/2021

e29751

Aprovado em: 17/10/2021

https://doi.org/10.47820/recima21.v2i9.751

\section{RESUMO}

As temperaturas extremas do ar apresentam tendências de variações diversificadas ao longo do tempo e possuem variações anuais quanto às suas características. A análise de períodos curtos, desde que realizados para anos sucessivos de no mínimo dez anos, proporciona avaliar o comportamento do clima da área. Objetiva-se analisar, temporal e espacialmente, as tendências climáticas anuais e estacionais, da temperatura máxima, média e mínima do ar em São Bento do Una - PE, no período de 1950-2016, bem como discutir possíveis causas. Os dados de temperatura máxima, média e mínima do ar foram estimados pelo software Estima_T, do período 1950-2016, e os referidos dados foram divididos a cada 10 anos. Utilizou-se da estatística básica para calcular as médias e outros parâmetros relevantes ao desenvolvimento do estudo. Os dados e as variabilidades de suas oscilações na temperatura do ar auxiliam às tomadas de decisões no meio agropecuário, urbano e rural em múltiplas áreas do conhecimento. A temperatura média é a representação da variabilidade e suas oscilações das temperaturas extremas, qualquer variabilidade nestes elementos as temperaturas médias se adequam as suas oscilações e decorrem dos sistemas sinóticos atuantes na época do período chuvoso e seco tal como dos impactos no meio ambiente e a sua elevação em relação ao nível do mar. Variabilidades climáticas registradas em escala global, regional e local foram constatadas na área estudada, no período de sessenta e seis anos de dados. Espera-se que essas informações possam subsidiar pesquisadores e tomadores de decisão em diversas áreas de atividade, especialmente na agricultura e avicultura.

PALAVRAS-CHAVES: Variáveis atmosféricas. Variabilidade climática. Componentes (a)bióticos.

\begin{abstract}
Extreme air temperatures show diversified variations trends over time and have annual variations in their characteristics. The analysis of short periods, provided that they are carried out for successive years of at least ten years, provides an assessment of the climate behavior of the area. The objective is to analyze, temporally and spatially, the annual and seasonal climate trends, of the maximum, average and minimum air temperature in São Bento do Una - PE, in the period 1950-2016, as well as to discuss possible causes. The Estima_T software estimated the maximum, mean and minimum air temperature data for the 1950-2016 period, and these data were divided every 10 years. Basic statistics were used to calculate means and other parameters relevant to the development of the study. The data and the variability of air temperature fluctuations help decision-making in the agricultural, urban and rural environment in multiple areas of knowledge. The average temperature is the representation of the variability and its oscillations of extreme temperatures, any variability in these elements the mean
\end{abstract}

\footnotetext{
1 Universidade Federal de Campina Grande - UFCG

2 Universidade Federal de Campina Grande - UFCG

3 Universidade Federal Rural de Pernambuco - UFRPE

${ }^{4}$ Universidade Federal Rural de Pernambuco - UFRPE

5 Universidade Federal Rural de Pernaumbuco - UFRPE

6 Universidade Estácio de Sá, Brasil - Graduando em Geografia Instituição: Universidade Estácio de Sá - Polo Recife

7 Universidade Federal Rural de Pernaumbuco - UFRPE
} 


\section{RECIMA21 - REVISTA CIENTÍFICA MULTIDISCIPLINAR ISSN 2675-6218} TEMPERATURAS DECADAIS EXTREMAS E MÉDIA EM SÃO BENTO DO UNA- PERNAMBUCO, BRASIL
Raimundo Mainar de Medeiros, Luciano Marcelo Falle Saboya, Manoel Vieira de França, Romildo Morant de Holanda, Fernando Cartaxo Rolim Neto, Wagner Rodolfo de Araújo, João Carlos Montenegro Coutinho Junior

temperatures fit their oscillations and result from the synoptic systems acting in the rainy and dry season, as well as from the impacts on the environment and its elevation in relation to sea level. Climatic variables registered on a global, regional and local scale were found in the studied area, in a period of sixty-six years of data. It is hoped that this information can support researchers and decision makers in various areas of activity, especially in agriculture and poultry.

KEYWORDS: Atmospheric variables. Climate variability. (A)biotic components.

\section{INTRODUÇÃO}

A temperatura do ar expressa de maneira simples a energia contida no meio. No decorrer de um dia a energia a disposição do ambiente oscila entre dois valores extremos, ou seja, entre a temperatura mínima e a máxima. Como essa energia vai de um extremo ao outro, ela atua em processos de contínuo estimulo aos processos fisiológicos vitais nos seres vivos, tais como sobre o desenvolvimento e crescimento das espécies vegetais, como: transpiração, respiração, germinação, crescimento, floração e frutificação. Em cada estágio de desenvolvimento da planta existem faixas adequadas de temperaturas para seu perfeito desenvolvimento de acordo com Costa et al. (2011). Além do mais o ciclo diário da temperatura predomina no conforto térmico e na adequação do ser humano e animal em determinados locais.

De acordo com as estimativas do Centro de Monitoramento de Deslocamento Interno (IDMC, 2017), até 2050 o número de refugiados por desastres climáticos chegará a 1 bilhão de pessoas. Recentemente, registraram-se deslocamentos por desastres climáticos - e eles não são poucos: algo em torno de 25 milhões de pessoas já deixaram seus países devido a problemas ambientais como secas, inundações, tempestades e incêndios florestais.

É consenso entre os cientistas da área de meteorologia e afins que as tendências climáticas observadas, especialmente a partir da segunda metade do século $\mathrm{XX}$, aumentaram significativamente. Segundo o Painel Intergovernamental de Mudanças Climáticas (Intergovernmental Panel on Climate Change - IPCC), essas tendências observadas no passado recente apresentam alta probabilidade de continuarem no mesmo sentido no século XXI (IPCC, 2007; IPCC, 2014).

Nas últimas décadas, as alterações climáticas e suas consequências para a humanidade tem sido uma das maiores preocupações de cientistas de todo o mundo. Principalmente no tocante aos fatores responsáveis pela variabilidade climática, que vêm se acentuando desde meados do século XX. As atividades humanas são, na visão de alguns pesquisadores, as responsáveis por parte destas mudanças. Entretanto, deve-se levar em consideração uma possível variabilidade climática natural, uma vez que a magnitude do sinal associada a ela nos registros climáticos existentes, ainda não foi bem determinada (IPCC, 1996; IPCC, 2001).

Medeiros et al. (2012) calcularam a temperatura do ar média diária com o emprego de diferentes metodologias para os municípios de Parnaíba, Picos e Gilbués localizados respectivamente na área litorânea, na região central e pertencente à região semiárida, em terras do cerrado e 


\section{RECIMA21 - REVISTA CIENTÍFICA MULTIDISCIPLINAR ISSN 2675-6218}

TEMPERATURAS DECADAIS EXTREMAS E MÉDIA EM SÃo BENTO DO UNA- PERNAMBUCO, BRASIL inar de Medeiros, Luciano Marcelo Falle Saboya, Manoel Vieira de França, Romildo Morant de Holanda, Fernando Cartaxo Rolim Neto, Wagner Rodolfo de Araújo, João Carlos Montenegro Coutinho Junior

desertificada do Estado do Piauí Utilizaram cinco métodos para o cálculo da temperatura média diária do ar, adotando como padrão o recomendado pelo Instituto Nacional de Meteorologia (INMET). Os quatro métodos avaliados em relação ao padrão possuíram desempenho classificado como "Muito bom e Ótimo", com índice de confiança variando entre 0,83 e 0,98. Os resultados ainda indicam que nas condições climáticas da região de estudo os quatro métodos avaliados em relação ao padrão (INMET) podem ser utilizados nas estimativas das temperaturas médias diárias do ar.

Nogueira et al. (2012) e Correia et al. (2011) concordam que a temperatura do ar se destaca entre as variáveis atmosféricas mais utilizadas no desenvolvimento de estudos de impactos ambientais com mudanças nos processos meteorológicos e hidrológicos.

Matos et al. (2015) utilizaram dados de temperatura do ar mensais para o município de Barbalha - Ceará e demonstraram que a elevação e a latitude são as variáveis fisiográficas que explicam melhor a variação da temperatura do ar anual e que as variabilidades da temperatura média decorrem dos sistemas sinóticos atuantes na época do período chuvoso ou seco tal como dos impactos no meio ambiente.

Medeiros et al. (2014) estudando as variabilidades da temperatura máxima, umidade relativa e precipitação para Brasília, notaram variabilidades nestes elementos climáticos com tendências a mudanças e afirmam que a redução nos índices de umidade e as taxas de aumentos na temperatura e precipitações são decorrentes dos efeitos locais os quais pode estar relacionado com o aumento da temperatura, que faz com que se tenha uma maior evaporação e consequentemente maior incidência pluvial.

Nogueira et al. (2012) e Correia et al. (2011) mostraram que as variabilidades das temperaturas do ar se destacam entre as variáveis atmosféricas mais utilizadas no desenvolvimento de estudos de impactos ambientais com mudanças nos processos meteorológicos e hidrológicos.

Silva (2000) afirmou que na região tropical, em boa parte do ano, a temperatura do ar, entre outros elementos ambientais, pode gerar estresse nos animais, que buscam se ajustar, aumentando a dissipação de calor por meio principalmente da termólise cutânea e da respiratória.

Nos dias atuais ocorrem muitas discussões sobre as flutuabilidades das temperaturas do ar (máxima, média e mínima) em todo o mundo, como possível aumento do efeito estufa provocado por ações antropogênicas. Praticamente todos os dados no mundo até algumas décadas atrás eram obtidos por meios convencionais e poucas estações operavam com dados durante as 24 horas do dia. Daí a necessidade de se trabalhar com os cálculos das temperaturas extremas e médias, utilizando seus valores estimados por software.

Objetiva-se analisar, temporal e espacialmente, as tendências climáticas anuais e estacionais, da temperatura máxima, média e mínima do ar por décadas e seu comparativo com a média climática no município de São Bento do Una - PE, no período de sessenta e seis anos (1950-2016), bem como discutir possíveis causas. Espera-se que essas informações possam subsidiar pesquisadores e tomadores de decisão em diversas áreas de atividade, especialmente na agropecuária e avicultura. 


\section{RECIMA21 - REVISTA CIENTÍFICA MULTIDISCIPLINAR ISSN 2675-6218}

TEMPERATURAS DECADAIS EXTREMAS E MÉDIA EM SÃO BENTO DO UNA- PERNAMBUCO, BRASIL Raimundo Mainar de Medeiros, Luciano Marcelo Falle Saboya, Manoel Vieira de França, Romildo Morant de Holanda, Fernando Cartaxo Rolim Neto, Wagner Rodolfo de Araújo, João Carlos Montenegro Coutinho Junior

\section{MATERIAL E MÉTODOS}

São Bento do Una localiza-se na mesorregião Agreste e na Microrregião do Vale do Ipojuca no Estado de Pernambuco, limitando-se a norte com Belo Jardim, a sul com Jucati, Jupi e Lajedo, a leste com Cachoeirinha, e a oeste com Capoeiras, Sanharó e Pesqueira.

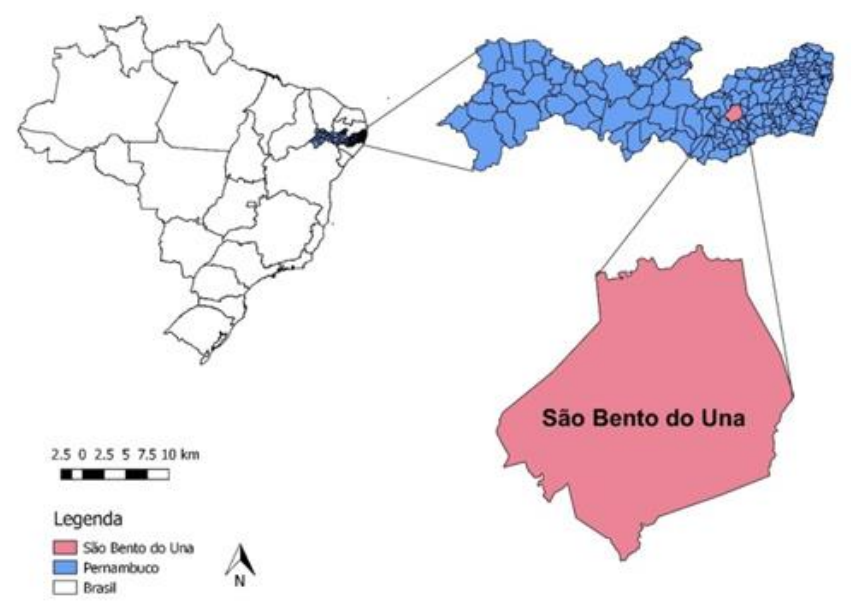

Figura 1. Localização do município de São Bento do Una no estado do Pernambuco.

Fonte: Medeiros, (2021).

A área municipal ocupa $719,15 \mathrm{~km}^{2}$ e representa $0.72 \%$ do Estado de Pernambuco. A sede do município tem altitude de 614 metros e coordenadas geográficas de $08^{\circ} 31^{\prime} 22^{\prime \prime}$ de latitude sul e $36^{\circ} 06^{\prime} 40^{\prime \prime}$ de longitude oeste. Com população estimada de 58.251 habitantes com densidade demográfica de 74,03 hab/.km²

São Bento do Una está inserido na unidade geoambiental do Planalto da Borborema, formada por maciços e outeiros altos, com altitude variando entre 650 e 1.000 metros. Ocupa uma área de arco que se estende do sul de Alagoas até o Rio Grande do Norte. O relevo é geralmente movimentado, com vales profundos e estreitos dissecados. Com respeito à fertilidade dos solos é bastante variada, com certa predominância de média para alta.

A área da unidade é recortada por rios perenes, porém de pequena vazão e o potencial de água subterrânea é baixo. A vegetação desta unidade é formada por Florestas Subcaducifólica e Caducifólica, próprias das áreas agrestes.

Segundo a classificação climática de Köppen (1928) São Bento do Una tem o clima As Tropical Chuvoso, com verão seco, esta classificação (MEDEIROS, et al., 2018; ALVARES et al., 2014).

A quadra chuvosa se inicia em fevereiro com chuvas de pré-estação (chuvas de pré-estação são as que ocorrem antes da quadra chuvosa) com seu término ocorrendo no final do mês de agosto 


\section{RECIMA21 - REVISTA CIENTÍFICA MULTIDISCIPLINAR ISSN 2675-6218}

TEMPERATURAS DECADAIS EXTREMAS E MÉDIA EM SÃo BENTO DO UNA- PERNAMBUCO, BRASIL Raimundo Mainar de Medeiros, Luciano Marcelo Falle Saboya, Manoel Vieira de França, Romildo Morant de Holanda, Fernando Cartaxo Rolim Neto, Wagner Rodolfo de Araújo, João Carlos Montenegro Coutinho Junior

e podendo se prolongar até a primeira quinzena de setembro. $O$ trimestre chuvoso centra-se nos meses de maio, junho e julho e os seus meses seco ocorrem entre outubro, novembro e dezembro. Os fatores provocadores de chuvas no município são a contribuição da Zona de Convergência Intertropical (ZCIT), formação dos vórtices ciclônicos de altos níveis (VCAS), contribuição dos ventos alísios de nordeste no transporte de vapor e umidade os quais condensam e forma nuvens provocando chuvas de moderadas a fortes intensidades, linhas de instabilidades, orografia e suas contribuições locais e regionais (MEDEIROS, 2016).

Utilizaram-se valores da temperatura extremas e média do ar estimadas pelo software Estima T (CAVALCANTI, et al., 1994; CAVALCANTI, et al., 2006). O Estima_T é um software para fazer estimativas de temperaturas do ar na Região do Nordeste Brasileiro (NEB). Determinaram-se os coeficientes da função quadrática para as temperaturas média, máxima e mínima mensal em função das coordenadas locais: longitude, latitude e altitude (CAVALCANTI, et al., 2006) dada por:

$$
\mathrm{T}=\mathrm{C}_{0}+\mathrm{C}_{1} \lambda+\mathrm{C}_{2} \varnothing+\mathrm{C}_{3} \mathrm{~h}+\mathrm{C}_{4} \lambda^{2}+\mathrm{C}_{5} \emptyset^{2}+\mathrm{C}_{6} \mathrm{~h}^{2}+\mathrm{C}_{7} \lambda \varnothing+\mathrm{C}_{8} \lambda \mathrm{h}+\mathrm{C}_{9} \varnothing \mathrm{h}
$$

Em que:

$\mathrm{C}_{0}, \mathrm{C}_{1}, \ldots ., \mathrm{C}_{9}$ são as constantes;

$\lambda, \lambda^{2}, \lambda \varnothing, \lambda \mathrm{h}$ longitude;

$\varnothing, \varnothing^{2}, \lambda \varnothing$ latitude;

$\mathrm{h}, \mathrm{h}^{2}, \lambda \mathrm{h}, \varnothing \mathrm{h}$ altura.

Os dados obtidos pelo software Estima-T correspondem ao período de 1950-2016, foram gerados por planilhas eletrônicas e realizam-se os cálculos básicos estatísticos para o desenvolvimento deste trabalho. Os dados de temperatura máxima, média e mínima do ar foram estimados pelo software Estima_T do período 1950-2016 e dividiu-se os referidos dados a cada 10 anos. Utilizou-se da estatística básica para calcular as médias e outros parâmetros relevantes ao desenvolvimento do estudo.

\section{RESULTADOS E DISCUSSÃO}

Neste estudo chama-se a atenção dos leitores para a metodologia de análise. Trabalhou-se com as médias decadais (1950-1959; 1960-1969; 1970-1979; 1980-1989; 1990-1999; 2000-2009 e 2010-2016), para as temperaturas máximas, médias e mínimas das referidas décadas e seu comparativo utilizou-se das medias das três temperaturas referentes ao período de 1950-2016.

O meio ambiente é constituído por um conjunto natural de componentes (a)bióticos em constantes e complexas interações. Nessas relações mútuas, o clima atua como resultante dessas influências recíprocas. Nos dias atuais a ocorrência de eventos atmosféricos extremos. As informações 


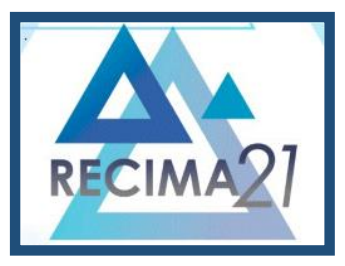

\section{RECIMA21 - REVISTA CIENTÍFICA MULTIDISCIPLINAR ISSN 2675-6218}

das variáveis meteorológicas são de fundamental importância para a elaboração de planejamento e de combate às graves consequências associada a esses elementos.

As temperaturas climáticas superaram as temperaturas da década de 1950-1959, nas oscilações das temperaturas máximas, médias e mínimas com aumento de $0,2^{\circ} \mathrm{C}$ em todos os meses e nos valores anuais (Figura 2). Estas variabilidades foram decorrentes dos sistemas meteorológicos de meso e microescala dos efeitos regional e local e da cobertura do solo visto que nesta década ainda não se tinha agricultura em expansão e a avicultura.

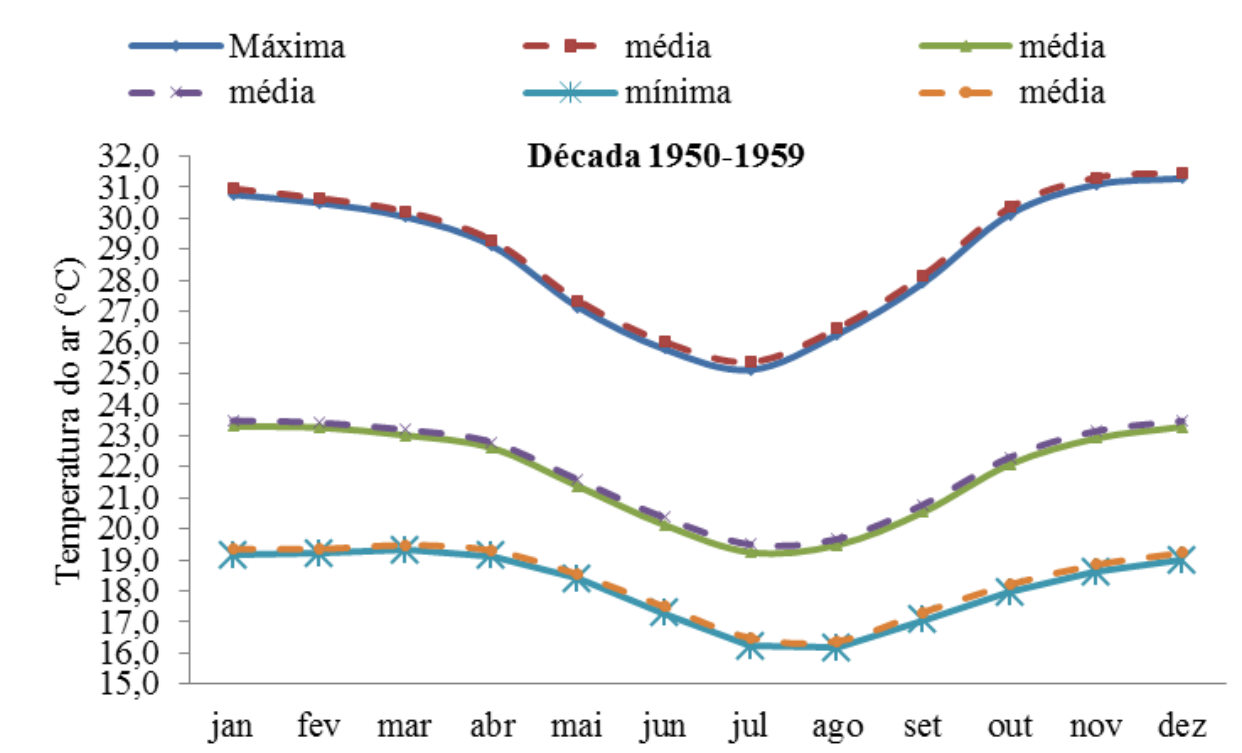

Figura 2. Temperatura decadais (1950-1959) e seu comparativo com a da série 1950-2016 em São Bento do Una - PE.

Fonte: Medeiros, (2021).

$\mathrm{Na}$ Figura 3 observam-se as variabilidades das temperaturas decadais (1960-1969) (MEDEIROS, et al., 2021) e seu comparativo com a média climática (1950-2016) no município de São Bento do Una - PE. As temperaturas médias da máxima, média e mínima superaram as oscilações das suas respectivas temperaturas decendial (1960-1969).

As variabilidades das temperaturas mínimas foram as que mais sofreram oscilações tais variabilidades estão em conformidade com o estudo de Rossato (2011). 


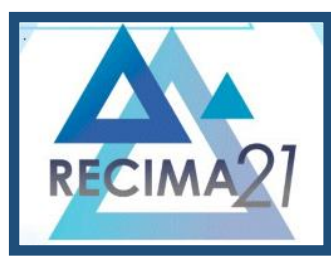

\section{RECIMA21 - REVISTA CIENTÍFICA MULTIDISCIPLINAR ISSN 2675-6218}

TEMPERATURAS DECADAIS EXTREMAS E MÉdIA EM SÃo BENTO DO UNA- PERNAMBUCO, BRASIL Raimundo Mainar de Medeiros, Luciano Marcelo Falle Saboya, Manoel Vieira de França, Romildo Morant de Holanda, Fernando Cartaxo Rolim Neto, Wagner Rodolfo de Araújo, João Carlos Montenegro Coutinho Junior

$$
\begin{aligned}
& \text { máxima - - média média } \\
& \text { - }- \text { média mínima } \quad-\infty \text { média }
\end{aligned}
$$

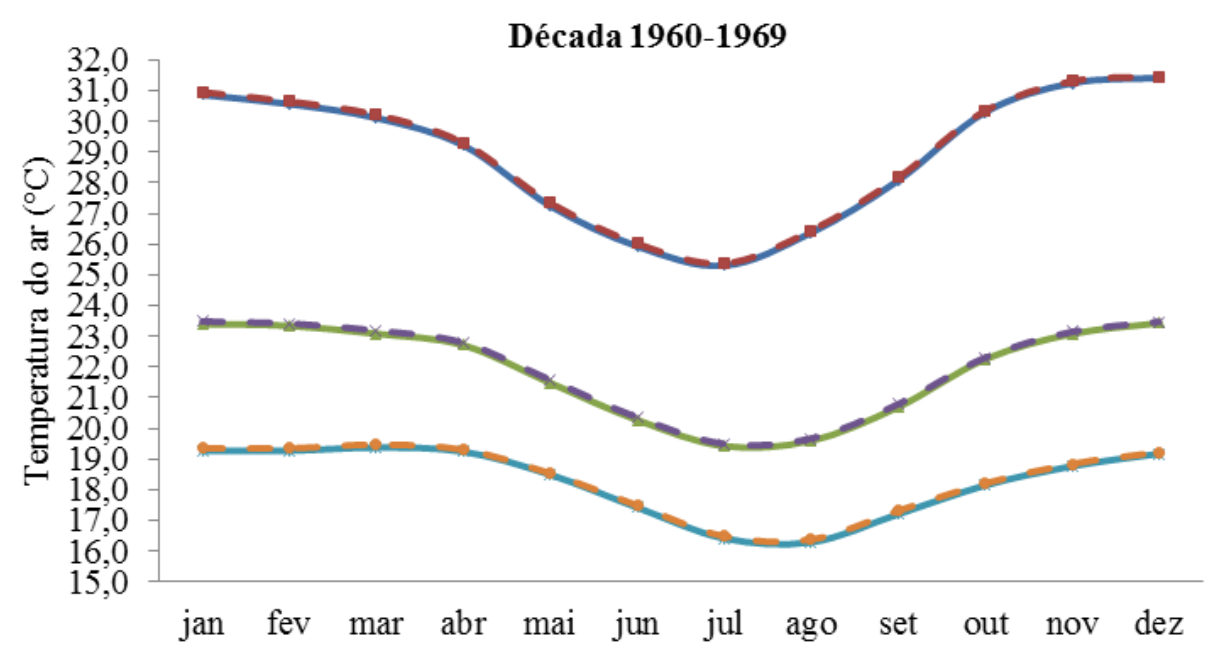

Figura 3. Temperatura decadais (1960-1969) e seu comparativo com a da série 1950-2016 em São Bento do Una - PE.

Fonte: Medeiros, (2021).

Araújo et al. (1998) observaram que, em média, as temperaturas mínimas vêm elevando-se, o que indica uma redução no grau de rigorosidade de inverno. $O$ autor ainda comenta que, em média, os invernos vêm registrando aquecimento, entretanto os eventos extremos da temperatura mínima têm ocorrido com menor frequência. Estes pesquisadores observaram que a amplitude dos invernos tem diminuído, pois existe um evidente aumento nas temperaturas mínimas em contraposição a um comportamento estacionário das temperaturas máximas.

Os meses de junho e julho registraram os menores valores da temperatura máxima, média e mínima para a década de 1970-1979 com redução de 0,2 $\mathrm{C}$. Os meses de outubro, novembro, dezembro e janeiro registraram-se os valores mais altos de temperatura. Destaca o mês de agosto nas oscilações da temperatura mínima que registrar o menor valor da série em estudo. Estas reduções no mês de agosto foram decorrentes das atividades dos sistemas locais (troca de calor, movimentos ascendentes, linha de instabilidade) seguidos de chuvas isoladas pela madrugada. 


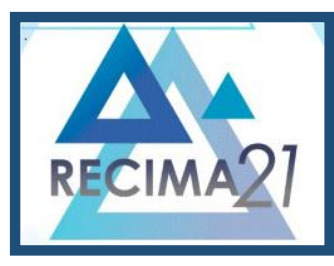

\section{RECIMA21 - REVISTA CIENTÍFICA MULTIDISCIPLINAR ISSN 2675-6218}

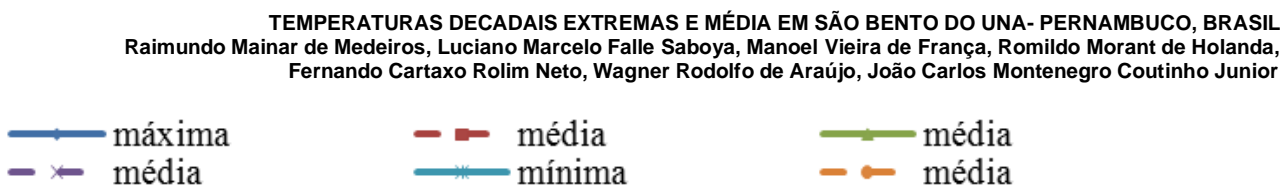

Década 1970-1979

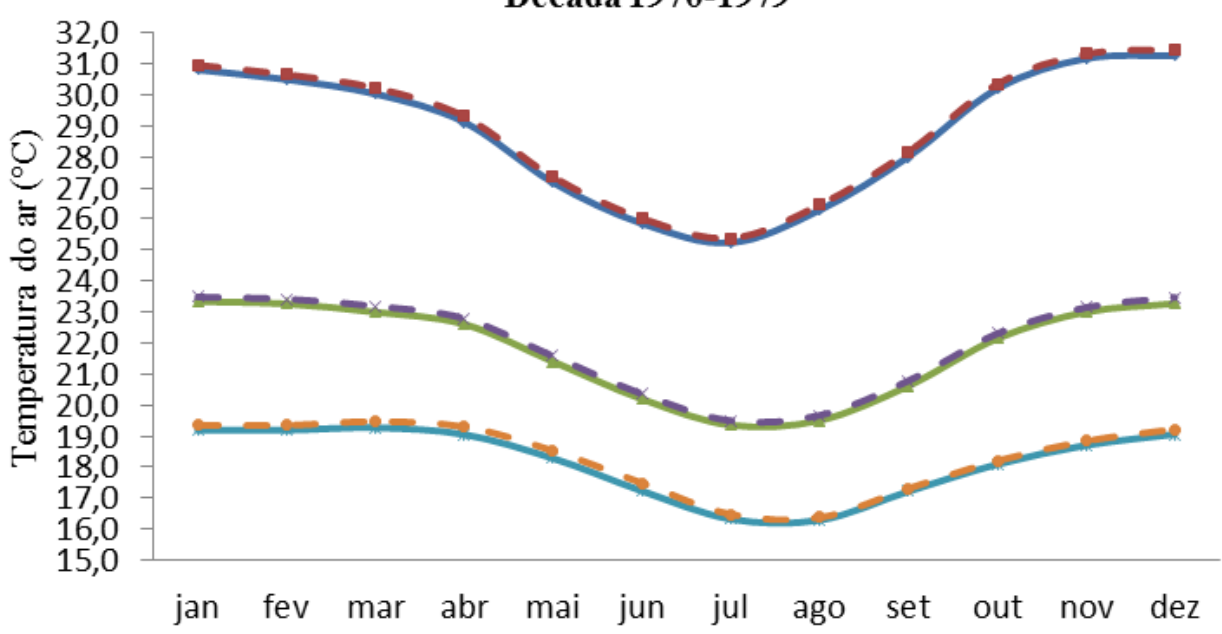

Figura 4. Temperatura decadais (1970-1979) e seu comparativo com a da série 1950-2016 em São Bento do Una - PE.

Fonte: Medeiros, (2021).

Campos (2010) afirmou que a variabilidade da temperatura do ar nas duas últimas décadas tem se destacado pelas elevadas anomalias de temperatura média global do ar (CLIMATE RESEARCH UNIT, 2009). Entre o período de 1961 a 1990, o ano 1998 foi o mais quente com $0,55^{\circ} \mathrm{C}$ acima da temperatura média anual. $\mathrm{O}$ ano de 2009 foi o sexto ano mais quente, com exceção dos anos de 1998, 2005, 2003, 2002, 2004.

Vincent et al. (2005) mostraram que existe a tendência expressiva de aumento da temperatura mínima, aumento de noites quentes e redução da amplitude diária em toda a América do Sul, especialmente no Sudeste da América do Sul, estes resultados vêm a corroborar com o presente estudo. As variabilidades das temperaturas máximas, médias e mínimas e seus comparativos com as médias climáticas para a década de 1980-1989 podem ser visualizados na Figura 5. 


\section{RECIMA21 - REVISTA CIENTÍFICA MULTIDISCIPLINAR ISSN 2675-6218}
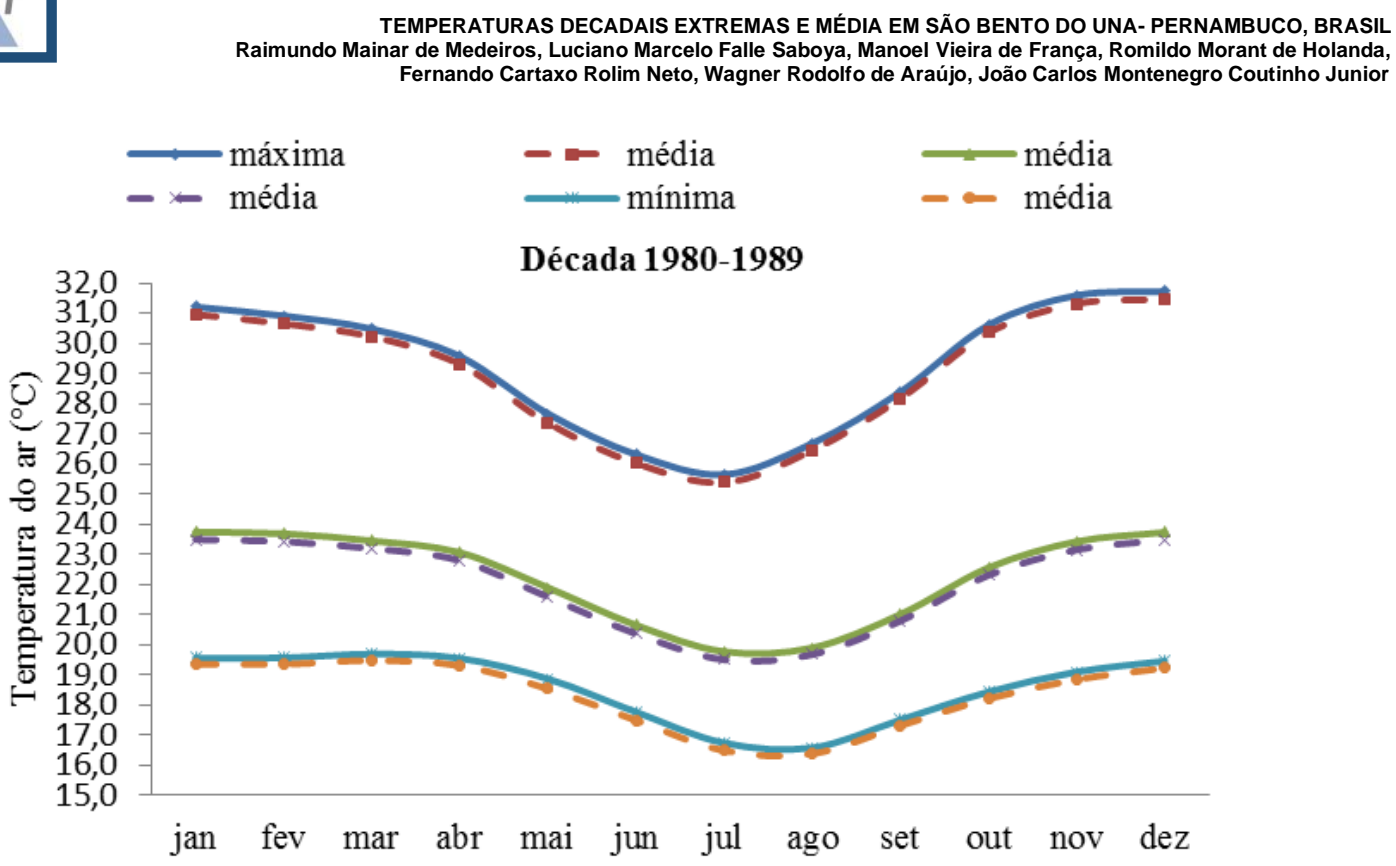

Figura 5. Temperaturas decadais (1980-1989) e seu comparativo com a série 1950-2016 em São Bento do Una - PE.

Fonte: Medeiros, (2021).

A temperatura máxima decadal oscilou entre $25,5^{\circ} \mathrm{C}$ em julho a $31,6^{\circ} \mathrm{C}$ em dezembro, a temperatura média anual da década foi de $29,1^{\circ} \mathrm{C}$. A temperatura climatológica da série 1950-2016 foi reduzida de $0,1^{\circ} \mathrm{C}$. A mesma redução foi registrada para as temperaturas médias e temperaturas mínimas

Melo et al. (2015) analisaram as variabilidades climáticas das temperaturas: máxima, média e mínima do estado de Pernambuco, enfocando tais variações como um meio para compreender futuras mudanças. Utilizaram dados de temperatura anual no período de 1960 a 1990. Afirmaram que o conhecimento do comportamento climático de uma região é importante para estudos de previsão do tempo e principalmente para o planejamento agropecuário, contribuindo com informações ao homem do campo para não realizar queimadas na hora do preparo das terras para o plantio, evitando desta forma que aumento do referido parâmetro ocorra. A delimitação de um período mais quente ou mais frio serve de alerta às autoridades federais, estaduais e municipais e tomadores de decisões, com objetivo de melhor planejamento. Os resultados encontrados no presente estudo corroboram com as discussões e com as variabilidades térmicas.

Na figura 6 observa-se as flutuações das temperaturas decadais (1990-1999) máximas, média e mínima e seu comparativo com a temperatura climatológica (1950-2016) para São Bento do Una $\mathrm{PE}$. As flutuações das temperaturas máximas, médias e mínimas das décadas fluíram acima das suas médias. As temperaturas máximas anuais dos três elementos analisados foram de: $29,2^{\circ} \mathrm{C}$ decadal e sua climatológica com $29^{\circ} \mathrm{C}$, as flutuações das temperaturas médias foram de $22,2^{\circ} \mathrm{C}$ e $22^{\circ} \mathrm{C}$ respectivamente as temperaturas mínimas e suas climatológicas foram de $18,6^{\circ} \mathrm{C}$ e $18,3^{\circ} \mathrm{C}$. 


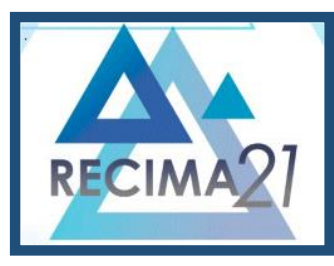

\section{RECIMA21 - REVISTA CIENTÍFICA MULTIDISCIPLINAR ISSN 2675-6218}

TEMPERATURAS DECADAIS EXTREMAS E MÉDIA EM SÃO BENTO DO UNA- PERNAMBUCO, BRASIL Raimundo Mainar de Medeiros, Luciano Marcelo Falle Saboya, Manoel Vieira de França, Romildo Morant de Holanda, Fernando Cartaxo Rolim Neto, Wagner Rodolfo de Araújo, João Carlos Montenegro Coutinho Junior

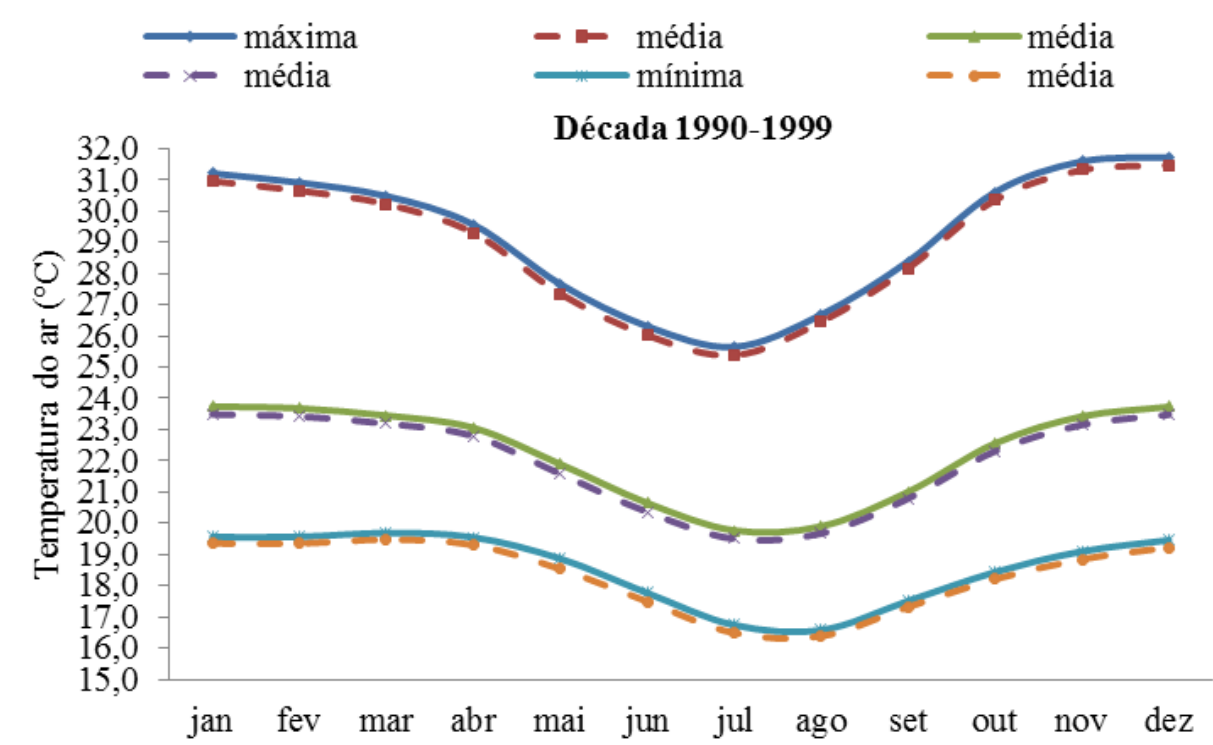

Figura 6. Temperatura decadais (1990-1999) e seu comparativo com a da série 1950-2016 em São Bento do Una - PE. - Fonte: Medeiros, (2021).

O IPCC (2007) afirma que onze dos doze anos anteriores a 2006 estão entre os anos mais quentes desde o período de 1850 , sendo que a temperatura média global aumentou linearmente aproximadamente $+0,1^{\circ} \mathrm{C}$ por década nos últimos 50 anos (1957 a 2006).

As oscilações das temperaturas máximas, médias e mínimas para a década de 2000-2009 e seus respectivos comparativos podem ser observadas na Figura 7. As temperaturas máximas e médias decadais superaram as médias climatológicas. A temperatura mínima apresentou oscilações de igualdade (junho a março) e aumento entre os meses de abril e maio na sua climatologia, estas flutuações estão em concordância com o (IPCC 2007; Marengo et al., 2006) corroborando com os resultados desta pesquisa.

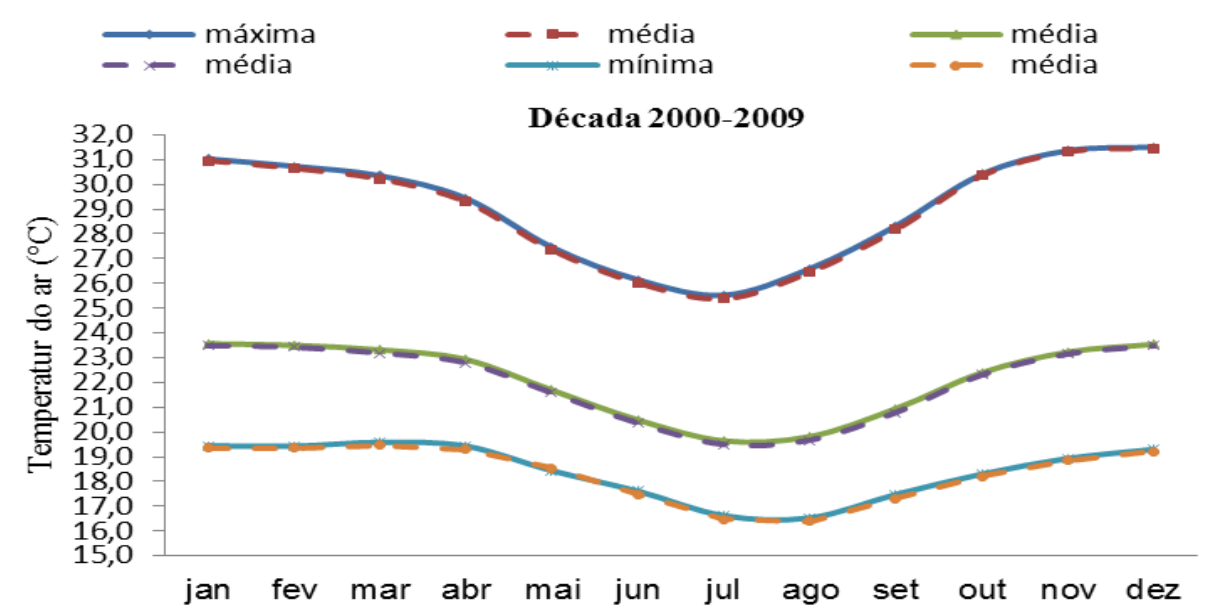

Figura 7. Temperatura decadais (2000-2009) e seu comparativo com a série 1950-2016 em São Bento do Una - PE. - Fonte: Medeiros, (2021). 


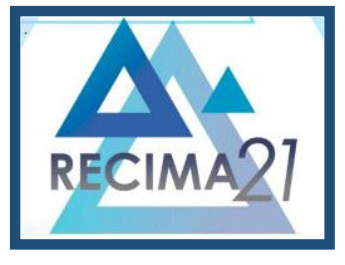

\section{RECIMA21 - REVISTA CIENTÍFICA MULTIDISCIPLINAR ISSN 2675-6218}

Na Figura 9 têm-se as flutuações da temperatura do período de 2010-2016 em São Bento do Una - PE. Observa-se que a temperatura máxima média e seu comparativo com a média do período de 1950-2016 mantiveram-se abaixo da média em todos os meses. No período de 2010-2016 a temperatura máxima anual foi de $28,9^{\circ} \mathrm{C}$ e suas oscilações mensais fluíram entre $25,3^{\circ} \mathrm{C}$ no mês de julho a $31,4^{\circ} \mathrm{C}$ em dezembro. A média climática do referido período em estudo oscilou com temperatura máxima anual de $29^{\circ} \mathrm{C}$ e suas oscilações mensais registraram-se com $25,4^{\circ} \mathrm{C}$ em julho a $31,5^{\circ} \mathrm{C}$ em dezembro. Na temperatura média das médias destacam-se suas flutuações mensais oscilando entre $19,4^{\circ} \mathrm{C}$ em julho a $23,4^{\circ} \mathrm{C}$ em dezembro e janeiro com temperatura média anual de $21,9^{\circ} \mathrm{C}$. $\mathrm{Na}$ variabilidade da temperatura média (1950-2016) a temperatura média foi de $22^{\circ} \mathrm{C}$ e as flutuações mensais registraram-se nos meses de julho com $19,5^{\circ} \mathrm{Ca} 23,5$ nos meses de dezembro e janeiro.

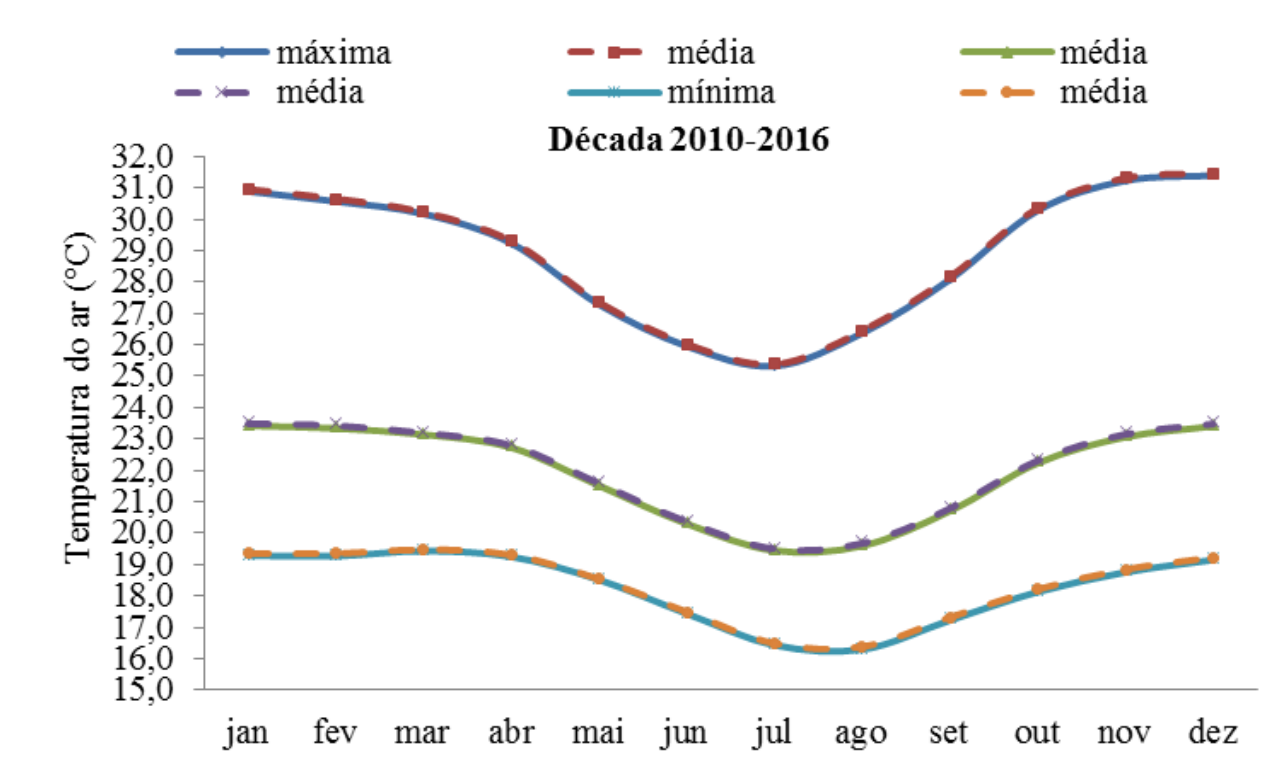

Figura 8. Temperatura decadais (2010-1016) e seu comparativo com a série 1950-2016 em São Bento do Una - PE.

Fonte: Medeiros, (2021).

Como o Nordeste do Brasil apresenta problemas com a falta e má distribuição dos postos meteorológicos, Medeiros et al. (2005), apud Bardin et al. (2010) estimaram e especializaram as temperaturas do ar (máxima, mínima e média) dessa região. Os coeficientes de determinação dos valores mensais e anuais de temperatura máxima, os coeficientes de determinação oscilaram de 0,85 (julho) a 0,52 (fevereiro) e, para os valores de temperatura mínima, de 0,85 (abril) a 0,64 (novembro) (BARDIN, et al., 2010). Para as temperaturas máximas médias mensais, a componente altitude teve significância de $5 \%$ de probabilidade, exceto nos meses maio, junho e julho. Porém, a latitude e a longitude não foram significativas em todos os meses, de acordo com estudos de Pezzopane et al. 


\section{RECIMA21 - REVISTA CIENTÍFICA MULTIDISCIPLINAR ISSN 2675-6218}

(2004), apud Berdin et al. (2010) que encontraram resultados aproximadamente semelhantes, e observaram que a variável longitude não foi significativa para o Estado do Espírito Santo.

\section{CONCLUSÕES}

Registram-se nas décadas de 1950, 1970, 1980, 1990 e 2000 aumento nas três temperaturas estudadas. Na década de 1960 ocorreram reduções nos três elementos. 2010 as temperaturas se equipararam com as temperaturas climáticas. As variabilidades observadas nas temperaturas decadais não estão interligadas as flutuações dos sistemas de meso e grande escala atuantes no período estudado.

Os dados e as variabilidades de suas oscilações na temperatura do ar auxiliam aos tomadores de decisões no meio agropecuário, urbano e rural em múltiplas áreas do conhecimento. A temperatura média é a representação da variabilidade e suas oscilações das temperaturas extremas, qualquer variabilidade nestes elementos as temperaturas médias se adequam as suas oscilações e decorrem dos sistemas sinóticos atuantes na época do período chuvoso e seco tal como dos impactos no meio ambiente e a sua elevação em relação ao nível do mar.

Variabilidades climáticas registradas em escala global, regional e local foram constatadas na área estudada, no período de sessenta e seis anos (1950-2016). As variabilidades de aumento na temperatura média do ar são devido à forte tendência de elevação da temperatura mínima do ar e das estabilidades nas suas oscilações mensais, anuais e decadais ora fluindo com reduções e aumentos podendo caracterizam tendência a possíveis mudanças climáticas.

A elevação e a latitude são as variáveis fisiográficas que explicam melhor a variação da temperatura do ar anual e que as variabilidades da temperatura média. A distribuição espacial da temperatura máxima, média e mínima apresentou variabilidade para ambos os meses e décadas estudadas, com variação de $0,1^{\circ} \mathrm{C}$ a $0,2^{\circ} \mathrm{C}$ nas suas décadas.

\section{REFERÊNCIAS}

ALVARES, C. A.; STAPE, J. L.; SENTELHAS, P. C.; GONÇALVES, J. L. M.; SPAROVEK, G. Köppen's climate classification map for Brazil. MeteorologischeZeitschrift, v. 22, p. 711-728, 2014.

ARAÚJO, G. P.; GONÇALVES, F. L. T. Análise climatológica preliminar de eventos extremos de frio no Parque Estadual das Fontes do Ipiranga - IAG/USP. In: Congresso Brasileiro de Agrometeorologia, 1998.

BARDIN, L.; JÚNIOR, M. J. P.; MORAES, J. F. L. Estimativa das temperaturas máximas e mínimas do ar para a região do Circuito das Frutas, SP. Revista Brasileira de Engenharia Agrícola e Ambiental, v. 14, n. 6, p. 618-624, 2010.

BURIOL, G. A. et al. Análise das temperaturas mínimas diárias no município de Santa Maria-RS. I Cálculo dos valores médios e da variabilidade, registro de valores extremos e comparação de periodicidade dessas temperaturas. In.: Anais... Congresso Brasileiro de Agrometeorologia, 5., 1987, Belém. O clima e o desenvolvimento rural brasileiro, Belém, 1987. p. 240-242. 

ISSN 2675-6218

CLIMATE RESEARCH UNIT. Global temperature record. Disponível em: http://www.cru.uea.ac.uk/cru/info/warming.

CAMPOS, C. G. C. Padrões climáticos atuais e futuros de temperatura do ar na região sul do Brasil e seus impactos nos cultivos de pêssego e nectarina. 2010. Tese (Doutorado em Meteorologia) - Instituto Nacional de Pesquisas Espaciais, São José dos Campos, 2010.

CAVALCANTI, E. P.; SILVA, V. P. R.; SOUSA, F. A. S. Programa computacional para a estimativa da temperatura do ar para a região Nordeste do Brasil. Revista Brasileira de Engenharia Agrícola e Ambiental, v. 10, n. 1, p. 140-147, 2006.

CAVALCANTI, E. P.; SILVA, E. D. V. Estimativa da temperatura do ar em função das coordenadas locais. In.: Anais... Congresso Brasileiro de Meteorologia, 8, 1994. Belo Horizonte, Belo Horizonte: SBMET, v.1, p. 154-157, 1994.

CORREIA, M. F.; SILVA, F. S.; SILVA, A. M. R. S.; SANTOS, E. P.; MOURA, M. S. B. Impacto da expansão agrícola na amplitude térmica diária em ambiente semiárido. Ciência e Natura, $v$. Suplementar, p. 311-314, 2011.

COSTA, T. S. A.; COSTA FILHO, J. F.; BARACHO, D. C.; SANTOS, T. S.; MARINHO, E. C. S. Análise da temperatura do ar em Areia - PB, em anos de ocorrência de "El Niño". Apresentado no XVII Congresso Brasileiro de Agrometeorologia - 18 a 21 de Julho de 2011 - SESC Centro de Turismo de Guarapari, Guarapari - ES. 2011.

IDMC. Centro de Monitoramento de Deslocamento Interno. Brasília: IDMC, 2017. p. 185.

INTERGOVERNAMENTAL PANEL ON CLIMATE CHANGE - IPCC. Climate Change 1996: impacts, adaptations and mitigation of climate change. Cambridge: Cambridge Univ. Press, 1996.

INTERGOVERNAMENTAL PANEL ON CLIMATE CHANGE - IPCC. Climate Change 2001: the scientific basis IPCC WG. Cambridge: Cambridge Univ. Press, 2001.

INTERGOVERNMENTAL PANEL ON CLIMATE CHANGE - IPCC. Climate Change 2007: The Physical Science Basis. Cambridge: Cambridge University Press, 2007.

IPCC. Intergovernmental Panel on Climate Change. 2014: Impacts, Adaptation, and Vulnerability. Part B: Regional Aspects. Working Group II Contribution to the Fifth. Assessment Report of the Intergovernmental Panel on Climate Change. Cambridge: Cambridge University Press, 2014.

KÖPPEN, W.; GEIGER, R. KLIMATE DER ERDE. Gotha: Verlag Justus Perthes. 1928. Wall-map $150 \mathrm{~cm} \times 200 \mathrm{~cm}$.

MARENGO, J.; SILVA, D. P. Mudanças climáticas globais e seus impactos nos recursos hídricos. In.: REBOUÇAS, A.; BRAGA, B.; TUNDISI, J. Em Águas Doces do Brasil: Capital Ecológico, Uso e Conservação, 2006. São Paulo: Editoras Escrituras, 2006. p. 63-109.

MATOS, R. M.; MEDEIROS, R. M; SILVA, P. F.; SABOYA, L. M. F. Flutuabilidade da temperatura média do ar anual, máximos e mínimos absolutos no município de Barbalha - CE, Brasil. In.: Anais Congresso Técnico Científico de Engenharia e da Agronomia. Congresso Técnico Científico de Engenharia e da Agronomia, 2015, Fortaleza - Ceará. 2015. v.1.

MEDEIROS, R. M.; HOLANDA, R. M.; VIANA, M. A.; SILVA, V. P. Climate classification in Köppen model for the state of Pernambuco - Brazil. Revista de Geografia (Recife). v.35, p.219 - 234, 2018. 


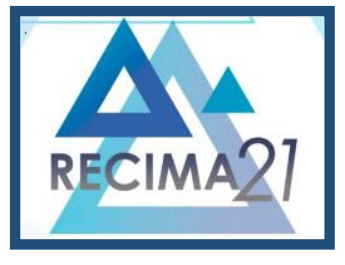

\section{RECIMA21 - REVISTA CIENTÍFICA MULTIDISCIPLINAR ISSN 2675-6218}

MEDEIROS, R. M. Estudo agroclimático do Estado do Pernambuco. [S. I.: s.n.]: 2016. p. 150.

MEDEIROS, R. M.; BORGES, C. K.; GOMES FILHO, M. F. Variabilidade e Mudanças Climáticas em Séries de Temperatura Máxima do Ar, Umidade Relativa do Ar e Precipitação em Brasília-DF. Revista Brasileira de Geografia Física, v. 07, n. 02, p. 211-222, 2014.

MEDEIROS, R. M.; TAVARES, A. L.; KASSAR, C. B.; SILVA, J. A. S.; SILVA, V. P. R. Metodologias de cálculo da temperatura média diária do ar: aplicação para os municípios de Parnaíba, Picos e Gillbués, PI. Revista Brasileira de Agricultura Irrigada, v. 6, p. 283-295, 2012.

MEDEIROS, S. S.; CECÍLIO, R. A.; MELO JÚNIOR, J. C. F.; SILVA JÚNIOR, J. L. C. Estimativa e espacialização das temperaturas do ar mínimas, médias e máximas na região Nordeste do Brasil. Revista Brasileira de Engenharia Agrícola e Ambiental, v. 9, n. 2, p. 247-255, 2005.

MELO, V. S.; MEDEIROS, R. M; SOUSA, F. A. S. Flutuabilidade da temperatura máxima e mínima do ar mensal e anual de 10 municípios do estado de Pernambuco. In.: Anais Congresso Técnico Científico de Engenharia e da Agronomia Congresso Técnico Científico de Engenharia e da Agronomia, 2015, Fortaleza - Ceará, v.1. 2015.

NOGUEIRA, V. F. B.; CORREIA, M. F.; NOGUEIRA, V. S. Impacto do Plantio de Soja e do Oceano Pacífico Equatorial na Precipitação e Temperatura na Cidade de Chapadinha - MA. Revista Brasileira de Geografia Física, v. 5, p. 708-724, 2012.

PEZZOPANE, J. R. M.; SANTOS, E. A.; EleutÉRIO, M. M.; REIS, E. F.; SANTOS, A. R. Espacialização da temperatura do ar no Estado do Espírito Santo. Revista Brasileira de Agrometeorologia, v. 12, n. 1, p. 151-158, 2004.

ROSSATO, M. S. Os climas do Rio Grande do Sul: variabilidade, tendências e tipologia. 2011. 240 f. Tese (Doutorado) - Universidade Federal do Rio Grande do Sul. Instituto de Geociências. Programa de Pós-Graduação em Geografia, Porto Alegre, 2011.

SILVA, R. G. Introdução á bioclimatologia animal. São Paulo: Nobel, 2000.

VINCENT, L. A. et al. Observed trends in indices of daily temperature extremes in South America 19602000. Journal of Climate, Boston, v. 18, p. 5011-5023, dez. 2005. 